\title{
Prevalence and Risk Factors of Silent Brain Infarction in Patients with Aortic Stenosis
}

\author{
Ayaka Ito $^{a}$ Shinichi Iwata ${ }^{a}$ Soichiro Tamura ${ }^{a}$ Andrew T. Kim ${ }^{a}$ \\ Shinichi Nonin ${ }^{a}$ Sera Ishikawa ${ }^{a}$ Asahiro Ito $^{a}$ Yasuhiro Izumiya ${ }^{a}$ \\ Takato Abe $^{b}$ Toshihiko Shibata ${ }^{c}$ Minoru Yoshiyama ${ }^{a}$ \\ ${ }^{a}$ Department of Cardiovascular Medicine, Osaka City University Graduate School of \\ Medicine Osaka, Osaka, Japan; ${ }^{b}$ Department of Neurology, Osaka City University Graduate \\ School of Medicine Osaka, Osaka, Japan; ' Department of Cardiovascular Surgery, Osaka \\ City University Graduate School of Medicine Osaka, Osaka, Japan
}

\section{Keywords}

Aortic stenosis - $\mathrm{CHA}_{2} \mathrm{DS}_{2}$-VASc score $\cdot$ Chronic kidney disease - Echocardiography · Silent brain infarction

\begin{abstract}
Introduction: Silent brain infarction (SBI) is an independent risk factor for subsequent symptomatic stroke in the general population. Although aortic stenosis (AS) is also known to be associated with an increased risk of future symptomatic stroke, little is known regarding the prevalence and risk factors for SBI in patients with AS. Methods: The study population comprised 83 patients with severe AS with no history of stroke or transient ischemic attack and paralysis or sensory impairment (mean age $75 \pm 7$ years). All patients underwent brain magnetic resonance imaging to screen for $\mathrm{SBI}$ and multidetector-row computed tomography to quantify the aortic valve calcification (AVC) volume. Comprehensive transthoracic and transesophageal echocardiography were performed to evaluate left atrial (LA) abnormalities, such as LA enlargement, spontaneous echo contrast, or abnormal LA appendage emptying velocity $(<20$ $\mathrm{cm} / \mathrm{s}$ ), and complex plaques in the aortic arch. Results: SBI was detected in 38 patients (46\%). Multiple logistic regression analysis indicated that $\mathrm{CHA}_{2} \mathrm{DS}_{2}-\mathrm{VASc}$ score and estimated glomerular filtration rate (eGFR) were independently associated with SBI $(p<0.05)$, whereas LA abnormalities and AVC volume were not. When patients were divided into 4 groups according to $\mathrm{CHA}_{2} \mathrm{DS}_{2}$-VASc score and eGFR, the group with a higher $\mathrm{CHA}_{2} \mathrm{DS}_{2}$-VASc score $(\geq 4)$ and a lower eGFR $\left(<60 \mathrm{~mL} / \mathrm{min} / 1.73 \mathrm{~m}^{2}\right)$ had a greater risk of SBI than the other groups $(p<0.05)$. Conclusion: These findings indicate that $A S$ is associated with a high prevalence of $S B I$, and that the $\mathrm{CHA}_{2} \mathrm{DS}_{2}$-VASc score and eGFR are useful for risk stratification.




\section{Cerebrovascular Diseases Extra}

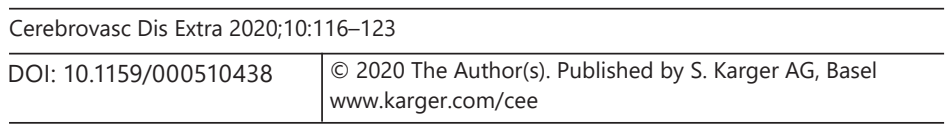

Ito et al.: Silent Brain Infarction in Patients with Aortic Stenosis

\section{Introduction}

Aortic stenosis (AS) is the most prevalent valvular heart disease; in the population aged $\geq 75$ years, the prevalence is $12.4 \%$ and that of severe AS is $3.4 \%$. The number of patients with AS is expected to increase 2- to 3 -fold over the next 50 years, owing to the aging population [1]. Since AS is associated with an approximately 2 -fold increased risk of ischemic stroke [2], which is linked to disability, poor prognosis, and higher health care costs, proper management is essential in daily patient care. The identification of promising preventive or therapeutic strategies for stroke could allow early intervention, and consequently improve the long-term ability of patients to carry out the activities of daily living, their quality of life, and the mortality rate.

Silent brain infarction (SBI), defined as a cerebral infarction that is incidentally detected by brain imaging in the absence of stroke-like clinical symptoms, has traditionally been considered a relatively benign degenerative change. However, this view changed recently when SBI was found to be associated with a 2- to 4 -fold increased risk of future symptomatic stroke [3] and a 2- to 3-fold increased risk of cognitive decline [4]. Despite this, no study to date has elucidated the prevalence and risk factors for SBI in patients with AS. The identification of modifiable risk factors associated with SBI would enable physicians to provide optimal personalized treatment for each AS patient to prevent SBI, consequently reduce future symptomatic stroke or dementia, and further support the maintenance of a better quality of life.

Therefore, the aim of this study was to elucidate the prevalence and associated risk factors for SBI in patients with AS.

\section{Materials and Methods}

\section{Study Population}

We conducted this cross-sectional study of prospectively collected data at the Osaka City University Hospital. We initially included 99 patients with severe AS, and no history of stroke or transient ischemic attack and paralysis or sensory impairment, who were admitted to our hospital for AS assessment. Cardiologists took a careful history and performed a neurological examination. We excluded 10 patients with other valvular heart diseases ( $\geq$ moderate), 4 with a history of cardiovascular surgery, and 2 with carotid artery stenosis or a history of carotid artery stenting. Thus, 83 patients were eligible for inclusion in this study.

\section{Transthoracic Echocardiography}

Comprehensive transthoracic echocardiography (TTE) was performed to evaluate AS and left atrial (LA) volume [5]. The aortic valve area was calculated using the continuity equation method [6]. We measured LA volume using the biplane modified Simpson method in the apical 4- and 2-chamber views. LA volume was indexed to the body surface area [5]. We performed bilateral carotid artery ultrasonography to exclude patients with ulcerated or mobile carotid plaques or carotid stenosis.

\section{Transesophageal Echocardiography}

Comprehensive transesophageal echocardiography (TEE) was performed to evaluate LA abnormalities such as abnormal LA appendage (LAA) emptying velocity, spontaneous echo contrast, or LA thrombus, and complex plaques in the aortic arch. Spontaneous echo contrast was defined as a dynamic smoke-like signal that swirled slowly in a circular pattern within the LA and LAA, that was continuously present at standard gain-after-gain adjustment to 
exclude background noise [7]. LAA emptying velocity was assessed using pulsed-wave Doppler at $10 \mathrm{~mm}$ caudal to the LAA orifice, averaged over at least 6 cardiac cycles $[8,9]$. Complex plaques were defined as large plaques ( $>4 \mathrm{~mm}$ thick), plaques with ulceration, or plaques with mobile components [10].

\section{Brain Magnetic Resonance Imaging}

All patients underwent brain magnetic resonance imaging (MRI) to detect SBI using a superconducting magnet at a field strength of 1.5 or $3.0 \mathrm{~T}$ to produce proton-density T1- and T2-weighted and fluid-attenuated inversion recovery (FLAIR) images. These images were obtained in the axial plane with a slice thickness of $5 \mathrm{~mm}$ and an interslice gap of $1.5 \mathrm{~mm}$. SBI was defined as a lesion measuring $>3 \mathrm{~mm}$ that appeared hypointense on T1-weighted images and hyperintense on T2-weighted images according to an AHA/ASA scientific statement [11] and a systematic review [3]. FLAIR images were used to distinguish dilated Virchow-Robin spaces from infarcts based on the absence or presence, respectively, of a hyperintense rim around the suspected lesions [11]. Moreover, we took their location of T2 high intensity into consideration to distinguish SBI from other structures.

\section{Multidetector-Row Computed Tomography}

All patients underwent noncontrast multidetector-row computed tomography (CT). Aortic valve calcification (AVC) was defined as the presence of any calcified lesions within the aortic valve leaflets. AVC was quantified using Ziostation2 automated software (Ziosoft Inc., Tokyo, Japan), calculated using the Agatston method with the threshold for calcium detection set at 130 Hounsfield units (HU) $[12,13]$ and expressed in arbitrary units (AU). We defined a region of interest for AVC quantification from the basal annular plane to the leaflet tips, and then carefully excluded calcified lesions involving the mitral annulus, left ventricular outflow tract, sinotubular junction, coronary arteries, and wall of the ascending aorta.

\section{Statistical Analysis}

All statistical analyses were performed using EZR graphical user interface (Saitama Medical Center, Jichi Medical University, Saitama, Japan) for $R$ programming language $(R$ Foundation for Statistical Computing, Vienna, Austria). The distributions of clinical characteristics and echocardiographic variables were compared between patients with SBI and those without SBI. Intergroup comparisons were performed using Fisher's exact test or the $\chi^{2}$ test for categorical variables and unpaired Student's $t$ test for continuous variables. Variables with $p$ values $<0.05$ were selected for multiple logistic regression analysis. As the $\mathrm{CHA}_{2} \mathrm{DS}_{2}$-VASc score includes hypertension and arch plaques, only the $\mathrm{CHA}_{2} \mathrm{DS}_{2}$-VASc score was entered into the multivariate analysis to avoid multicollinearity. Multiple logistic regression analysis was performed to identify variables associated with the presence of SBI in patients with severe AS.

\section{Results}

\section{Clinical and Echocardiographic Variables Associated with SBI}

The patients' clinical and echocardiographic characteristics by SBI status are shown in Table 1. Of 83 patients with severe AS, SBI was observed in $38(46 \%)$. Hypertension $(p<$ $0.001)$, complex arch plaques ( $p=0.02)$, and $\mathrm{CHA}_{2} \mathrm{DS}_{2}$-VASc score $(p=0.004)$ were positively associated with SBI, but LA abnormalities, AVC volume, antiplatelet therapy, coronary artery disease, a history of percutaneous coronary intervention (PCI), and current blood pressure values were not. Estimated glomerular filtration rate (eGFR) was negatively associated with 
Table 1. Clinical and echocardiographic characteristics according to SBI status

\begin{tabular}{|c|c|c|c|c|}
\hline & $\begin{array}{l}\text { All patients } \\
(n=83)\end{array}$ & $\begin{array}{l}\text { SBI }(-) \\
(n=45)\end{array}$ & $\begin{array}{l}\text { SBI }(+) \\
(n=38)\end{array}$ & $p$ value \\
\hline Age, years & $75 \pm 7$ & $73 \pm 7$ & $76 \pm 6$ & 0.07 \\
\hline Male sex & $44(53)$ & $20(44)$ & $24(63)$ & 0.12 \\
\hline Congestive heart failure & 49 (59) & $26(58)$ & $23(61)$ & 0.83 \\
\hline Diabetes mellitus & $26(31)$ & $13(29)$ & $13(34)$ & 0.61 \\
\hline Hypertension & $62(75)$ & $27(60)$ & $35(92)$ & $<0.001$ \\
\hline Dyslipidemia & $46(55)$ & $25(56)$ & $21(55)$ & 1.00 \\
\hline eGFR, $\mathrm{mL} / \mathrm{min} / 1.73 \mathrm{~m}^{2}$ & $50 \pm 26$ & $57 \pm 22$ & $42 \pm 29$ & 0.006 \\
\hline Atrial fibrillation & $10(12)$ & $3(7)$ & $7(18)$ & 0.17 \\
\hline Antiplatelet therapy & $35(42)$ & $16(55)$ & $19(50)$ & 0.27 \\
\hline Coronary artery disease & $35(42)$ & $15(33)$ & $20(53)$ & 0.12 \\
\hline A history of PCI & $9(11)$ & $2(4)$ & 7 (18) & 0.07 \\
\hline Systolic blood pressure, mm Hg & $130 \pm 20$ & $130 \pm 19$ & $131 \pm 20$ & 0.86 \\
\hline Diastolic blood pressure, $\mathrm{mm} \mathrm{Hg}$ & $68 \pm 12$ & $69 \pm 14$ & $67 \pm 10$ & 0.42 \\
\hline Aortic valve area, $\mathrm{cm}^{2}$ & $0.70 \pm 0.15$ & $0.69 \pm 0.16$ & $0.71 \pm 0.12$ & 0.40 \\
\hline Left ventricular ejection fraction, $\%$ & $58 \pm 11$ & $58 \pm 13$ & $57 \pm 8$ & 0.45 \\
\hline $\mathrm{e}^{\prime}, \mathrm{cm} / \mathrm{s}$ & $4.2 \pm 1.5$ & $4.3 \pm 1.6$ & $4.0 \pm 1.2$ & 0.37 \\
\hline $\mathrm{E} / \mathrm{e}^{\prime}$ & $21 \pm 10$ & $21 \pm 8$ & $23 \pm 12$ & 0.31 \\
\hline Left atrial volume index, $\mathrm{mL} / \mathrm{m}^{2}$ & $46 \pm 10$ & $44 \pm 18$ & $50 \pm 20$ & 0.15 \\
\hline Spontaneous echo contrast & $9(11)$ & $3(7)$ & $6(16)$ & 0.29 \\
\hline LAA emptying velocity, $\mathrm{cm} / \mathrm{s}$ & $48 \pm 21$ & $49 \pm 20$ & $47 \pm 23$ & 0.67 \\
\hline Complex arch plaque & $23(28)$ & 7 (16) & $15(40)$ & 0.02 \\
\hline $\mathrm{CHA}_{2} \mathrm{DS}_{2}$-VASc score & $4.0 \pm 1.4$ & $3.6 \pm 1.5$ & $4.5 \pm 1.1$ & 0.004 \\
\hline Aortic valve calcium volume, $\mathrm{AU}$ & $3,711 \pm 2,135$ & $3,710 \pm 2,290$ & $3,712 \pm 1,969$ & 0.99 \\
\hline
\end{tabular}

Values express $n(\%)$ or mean \pm SD. AU, arbitrary units; eGFR, estimated glomerular filtration rate; LAA, left atrial appendage; PCI, percutaneous coronary intervention; SBI, silent brain infarction.

Table 2. Multiple logistic regression analysis for the prediction of SBI

\begin{tabular}{llll}
\hline & OR & $95 \%$ CI & $p$ value \\
\hline eGFR, mL/min/1.73 m ${ }^{2}$ & 0.98 & $0.96-1.00$ & 0.03 \\
CHA $_{2} \mathrm{DS}_{2}$-VASc score (per 1 point) & 1.58 & $1.08-2.30$ & 0.02 \\
\hline
\end{tabular}

OR, odds ratio; CI, confidence interval; eGFR, estimated glomerular filtration rate; SBI, silent brain infarction.

SBI $(p=0.006)$. Table 2 shows the clinical and echocardiographic variables associated with the presence of SBI and their odds ratios (ORs) obtained by multiple logistic regression analysis. Both eGFR (OR 0.98; 95\% confidence interval [CI] 0.96-1.00; $p=0.03$ ) and $\mathrm{CHA}_{2} \mathrm{DS}_{2}-$ VASc score (OR 1.58; 95\% CI 1.08-2.30; $p=0.02$ ) remained independently associated with the presence of SBI.

\section{Combination of eGFR and $\mathrm{CHA}_{2} \mathrm{DS}_{2}$-VASc Score for the Prediction of SBI}

All patients were divided into 4 groups according to $\mathrm{CHA}_{2} \mathrm{DS}_{2}$-VASc score and eGFR. $\mathrm{CHA}_{2} \mathrm{DS}_{2}$-VASc scores were divided into $\geq 4$ and $<4$, based on receiver-operating characteristics (ROC) curve analysis, which determined the optimal threshold $\mathrm{CHA}_{2} \mathrm{DS}_{2}$-VASc score for predicting SBI with $82 \%$ sensitivity and $51 \%$ specificity (area under the curve [AUC] 0.66). The groupings for eGFR were $\geq 60$ and $<60 \mathrm{~mL} / \mathrm{min} / 1.73 \mathrm{~m}^{2}$, based on the Kidney Disease 


\section{Cerebrovascular Diseases Extra}

\begin{tabular}{l|l}
\hline Cerebrovasc Dis Extra 2020;10:116-123 \\
\hline DOI: 10.1159/000510438 & $\begin{array}{l}\text { @ 2020 The Author(s). Published by S. Karger AG, Basel } \\
\text { www.karger.com/cee }\end{array}$ \\
\hline
\end{tabular}

Ito et al.: Silent Brain Infarction in Patients with Aortic Stenosis
Fig. 1. Combination of $\mathrm{CHA}_{2} \mathrm{DS}_{2}$ VASc score and renal impairment for the prediction of SBI. eGFR, estimated glomerular filtration rate; SBI, silent brain infarction.

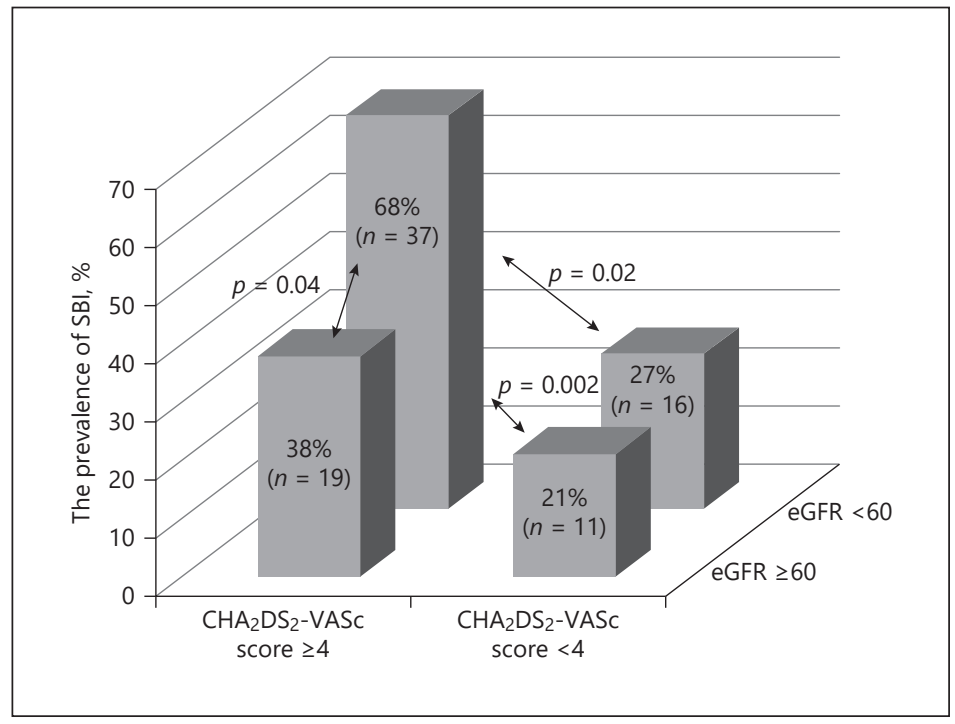

Improving Global Outcomes (KDIGO) 2012 guideline [14]. The group with a higher $\mathrm{CHA}_{2} \mathrm{DS}_{2^{-}}$ VASc score and a lower eGFR had a significantly higher prevalence of SBI than the other groups (Fig. 1).

\section{Discussion}

In this study, we observed a higher prevalence of SBI (46\%) in patients with severe AS than that reported for the general population $(10.7 \%)$ and patients with atrial fibrillation (AF) $(25 \%)[15,16]$. Moreover, we showed that $\mathrm{CHA}_{2} \mathrm{DS}_{2}$-VASc score and eGFR were significantly associated with SBI, but that LA abnormalities and AVC volume did not predict SBI. These results suggest that microembolization from LA abnormalities or a calcified aortic valve may not be major mechanisms of SBI in patients with AS. We also showed that the combination of $\mathrm{CHA}_{2} \mathrm{DS}_{2}$-VASc score and eGFR has an additional predictive value for SBI.

In our study, $\mathrm{CHA}_{2} \mathrm{DS}_{2}$-VASc score was significantly associated with $\mathrm{SBI}$ in patients with severe AS. This finding is consistent with those of previous studies showing the importance of $\mathrm{CHA}_{2} \mathrm{DS}_{2}$-VASc score in predicting SBI. For example, previous studies reported that $\mathrm{CHA}_{2} \mathrm{DS}_{2}$-VASc score was associated with SBI in the population with AF $[10,16]$. However, these studies were based on patients with $\mathrm{AF}$, and no studies to date have elucidated the association between $\mathrm{CHA}_{2} \mathrm{DS}_{2}$-VASc score and $\mathrm{SBI}$ in patients with AS. Based on our results, we speculate that the $\mathrm{CHA}_{2} \mathrm{DS}_{2}$-VASc score could also be useful for the prediction of cerebral small-vessel disease in patients with AS. The mechanism underlying this finding remains poorly understood. However, there are several mechanisms that could provide a connection between $\mathrm{CHA}_{2} \mathrm{DS}_{2}$-VASc score and SBI. Age, hypertension, diabetes mellitus [11], and complex plaques in the aortic arch [10] are all components of the $\mathrm{CHA}_{2} \mathrm{DS}_{2}$-VASc score reported to be associated with SBI. $\mathrm{CHA}_{2} \mathrm{DS}_{2}$-VASc score, which represents a patient's atherosclerotic burden, may therefore be useful for risk stratification of the early stage of cerebral damage in patients who have AS.

In our study, renal impairment was significantly associated with SBI in patients with AS. This finding is consistent with previous studies showing the importance of renal impairment in predicting SBI. Toyoda et al. [17] reported that chronic kidney disease (CKD) was asso- 
ciated with SBI in the general population. Similarly, several studies have shown positive associations between CKD stage and SBI in patients with CKD [18, 19]. However, no studies to date have elucidated the association between CKD and SBI in patients with AS. The mechanisms underlying our findings remain poorly understood. There are 2 mechanisms, however, that could explain the impact of renal impairment on SBI. First, injuries to the small vessels in both the kidneys and brain are reported to be mediated by a unique mechanism owing to how these vessels differ from other arteries, i.e., arteriosclerosis can result from exposure to greater pulsatile circumferential stress and longitudinal shear stress due to the need to maintain perfusion directly from the large arteries to the tissues [18, 20, 21]. Therefore, the presence of CKD may reflect the intrinsic severity of subclinical cerebral small-vessel occlusive disease. Second, renal impairment promotes endothelial dysfunction due to the secretion of uremic toxins, inflammation-related cytokines, and oxidative stress markers as well as the activation of the sympathetic nervous system. In support of this idea, renal impairment seems to lead to an increased risk of SBI occurrence in patients with AS $[17,22,23]$.

Although there is evidence of an association between SBI and LA abnormalities such as LA enlargement, LA thrombus, spontaneous echo contrast, and abnormal LAA emptying velocity in patients with nonvalvular AF [10], this relationship has not been confirmed in patients with AS. This difference might, in part, stem from the difference in SBI mechanisms (i.e., microembolization and small-vessel occlusive disease) in different populations. LA abnormalities are mainly linked to SBI due to microembolization resulting from LA blood stasis that is more specific to AF and may not reflect an atherosclerotic process. Thus, the predictive value of LA abnormalities for SBI, beyond atherosclerotic risk classification using $\mathrm{CHA}_{2} \mathrm{DS}_{2}$-VASc score or renal impairment, is limited in patients with AS.

Our study observed no significant association between AVC volume and SBI in patients with AS. This indicates that spontaneous cerebral embolization from a calcified aortic valve may not be a major cause of SBI in patients with AS. Similarly, Boon et al. [24] failed to show a significant association between AVC and SBI in a cohort of patients with AVC with or without stenosis. Conversely, Rodriguez et al. [25] succeeded in showing an association between SBI and aortic valve sclerosis; however, their observations cannot necessarily be extended to patients with AS because their study involved the general population aged $\geq 65$ years without any resultant outflow obstruction. Moreover, the methodology used in our study was more sensitive, assessing AVC quantitatively (AVC volume) as opposed to only qualitatively (i.e., according to the presence or absence of AVC as in the previous study).

Our study has several limitations. We used cross-sectional data, so first, we could not determine a causal relationship between $\mathrm{CHA}_{2} \mathrm{DS}_{2}$-VASc score or eGFR and SBI. Hence, prospective studies are necessary to assess whether $\mathrm{CHA}_{2} \mathrm{DS}_{2}$-VASc score and eGFR do indeed predict SBI in patients with severe AS, and second, could not perform a reliable analysis regarding prognosis. Prospective studies are needed to assess whether interventions for $\mathrm{CHA}_{2} \mathrm{DS}_{2}$-VASc score and eGFR (i.e., treatment for heart failure, hypertension, and diabetes or antiplatelet therapy in patients who have renal impairment and a higher $\mathrm{CHA}_{2} \mathrm{DS}_{2}$-VASc score) do indeed prevent SBI in patients with severe AS. Third, although there were no significant differences concerning a history of PCI, we could not completely rule out the possibility that SBI in our study may actually have been lesions associated with past PCI. Fourth, since the neurological assessment was performed by cardiologists, the prevalence of neurological disorders might have been underestimated. Fifth, as our population comprised patients with severe AS, the generalizability of our results to patients with all stages of AS is limited.

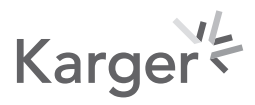




\section{Cerebrovascular Diseases Extra}

\begin{tabular}{l|l}
\hline Cerebrovasc Dis Extra 2020;10:116-123 \\
\hline DOI: 10.1159/000510438 & $\begin{array}{l}\text { ○ 2020 The Author(s). Published by S. Karger AG, Basel } \\
\text { www.karger.com/cee }\end{array}$ \\
\hline
\end{tabular}

Ito et al.: Silent Brain Infarction in Patients with Aortic Stenosis

\section{Conclusion}

AS is associated with a higher prevalence of SBI. Renal impairment and a higher $\mathrm{CHA}_{2} \mathrm{DS}_{2}-$ VASc score, but not LA abnormalities or AVC volume, are associated with SBI. The combination of renal impairment and $\mathrm{CHA}_{2} \mathrm{DS}_{2}$-VASc score has additional predictive value for SBI. These findings will be useful for risk stratification and may contribute to reducing future stroke or dementia and further supporting the maintenance of a better quality of life.

\section{Statement of Ethics}

This study was performed in line with the principles of the Declaration of Helsinki. Approval was granted by the Ethics Committee of the Osaka City University Graduate School of Medicine (ref. No. 3805). Informed consent was obtained from each patient included in the study.

\section{Conflict of Interest Statement}

The authors have no conflicts of interest to declare.

\section{Funding Sources}

There was no funding.

\section{Author Contributions}

A.I. prepared the manuscript; S.I. designed the study; A.K., S.T., S.N., S.I., and A.I. analyzed and interpreted data; Y.I, T.A., and T.S. performed critical revision of the article; M.Y. approved the final version of the manuscript for publication.

\section{References}

1 Osnabrugge RL, Mylotte D, Head SJ, Van Mieghem NM, Nkomo VT, LeReun CM, et al. Aortic stenosis in the elderly: disease prevalence and number of candidates for transcatheter aortic valve replacement: a metaanalysis and modeling study. J Am Coll Cardiol. 2013 Sep;62(11):1002-12.

2 Otto CM, Lind BK, Kitzman DW, Gersh BJ, Siscovick DS. Association of aortic-valve sclerosis with cardiovascular mortality and morbidity in the elderly. N Engl J Med. 1999 Jul;341(3):142-7.

3 Gupta A, Giambrone AE, Gialdini G, Finn C, Delgado D, Gutierrez J, et al. Silent Brain Infarction and Risk of Future Stroke: A Systematic Review and Meta-Analysis. Stroke. 2016 Mar;47(3):719-25.

4 Chen LY, Lopez FL, Gottesman RF, Huxley RR, Agarwal SK, Loehr L, et al. Atrial fibrillation and cognitive decline - the role of subclinical cerebral infarcts: the Atherosclerosis Risk in Communities Study. Stroke. 2014 Sep; 45(9):2568-74.

5 Lang RM, Badano LP, Mor-Avi V, Afilalo J, Armstrong A, Ernande L, et al. Recommendations for cardiac chamber quantification by echocardiography in adults: an update from the American Society of Echocardiography and the European Association of Cardiovascular Imaging. Eur Heart J Cardiovasc Imaging. 2015 Mar;16(3):23370.

6 Baumgartner H, Hung J, Bermejo J, Chambers JB, Edvardsen T, Goldstein S, et al. Recommendations on the Echocardiographic Assessment of Aortic Valve Stenosis: A Focused Update from the European Association of Cardiovascular Imaging and the American Society of Echocardiography. J Am Soc Echocardiogr. 2017 Apr; $30(4): 372-92$. 
7 Chimowitz MI, DeGeorgia MA, Poole RM, Hepner A, Armstrong WM. Left atrial spontaneous echo contrast is highly associated with previous stroke in patients with atrial fibrillation or mitral stenosis. Stroke. 1993 Jul; 24(7):1015-9.

8 Fatkin D, Kelly RP, Feneley MP. Relations between left atrial appendage blood flow velocity, spontaneous echocardiographic contrast and thromboembolic risk in vivo. J Am Coll Cardiol. 1994 Mar;23(4):961-9.

9 Zabalgoitia M, Halperin JL, Pearce LA, Blackshear JL, Asinger RW, Hart RG; Stroke Prevention in Atrial Fibrillation III Investigators. Transesophageal echocardiographic correlates of clinical risk of thromboembolism in nonvalvular atrial fibrillation. J Am Coll Cardiol. 1998 Jun;31(7):1622-6.

10 Sugioka K, Takagi M, Sakamoto S, Fujita S, Ito A, Iwata S, et al. Predictors of silent brain infarction on magnetic resonance imaging in patients with nonvalvular atrial fibrillation: A transesophageal echocardiographic study. Am Heart J. 2015 Jun;169(6):783-90.

11 Smith EE, Saposnik G, Biessels GJ, Doubal FN, Fornage M, Gorelick PB, et al.; American Heart Association Stroke Council; Council on Cardiovascular Radiology and Intervention; Council on Functional Genomics and Translational Biology; and Council on Hypertension. Prevention of Stroke in Patients with Silent Cerebrovascular Disease: A Scientific Statement for Healthcare Professionals From the American Heart Association/American Stroke Association. Stroke. 2017 Feb;48(2):e44-71.

12 Agatston AS, Janowitz WR, Hildner FJ, Zusmer NR, Viamonte M Jr, Detrano R. Quantification of coronary artery calcium using ultrafast computed tomography. J Am Coll Cardiol. 1990 Mar;15(4):827-32.

13 Linefsky J, Katz R, Budoff M, Probstfield J, Owens D, Takasu J, et al. Stages of systemic hypertension and blood pressure as correlates of computed tomography-assessed aortic valve calcium (from the Multi-Ethnic Study of Atherosclerosis). Am J Cardiol. 2011 Jan;107(1):47-51.

14 Chapter 1: definition and classification of CKD. Kidney Int Suppl (2011). 2013 Jan;3(1):19-62.

15 Das RR, Seshadri S, Beiser AS, Kelly-Hayes M, Au R, Himali JJ, et al. Prevalence and correlates of silent cerebral infarcts in the Framingham offspring study. Stroke. 2008 Nov;39(11):2929-35.

16 Ishikawa S, Sugioka K, Sakamoto S, Fujita S, Ito A, Norioka N, et al. Relationship between tissue Doppler measurements of left ventricular diastolic function and silent brain infarction in patients with non-valvular atrial fibrillation. Eur Heart J Cardiovasc Imaging. 2017 Nov;18(11):1245-52.

17 Toyoda G, Bokura H, Mitaki S, Onoda K, Oguro H, Nagai A, et al. Association of mild kidney dysfunction with silent brain lesions in neurologically normal subjects. Cerebrovasc Dis Extra. 2015 Feb;5(1):22-7.

18 Kobayashi M, Hirawa N, Yatsu K, Kobayashi Y, Yamamoto Y, Saka S, et al. Relationship between silent brain infarction and chronic kidney disease. Nephrol Dial Transplant. 2009 Jan;24(1):201-7.

19 Shima H, Ishimura E, Naganuma T, Ichii M, Yamasaki T, Mori K, et al. Decreased kidney function is a significant factor associated with silent cerebral infarction and periventricular hyperintensities. Kidney Blood Press Res. 2011;34(6):430-8.

20 O'Rourke MF, Safar ME. Relationship between aortic stiffening and microvascular disease in brain and kidney: cause and logic of therapy. Hypertension. 2005 Jul;46(1):200-4.

21 Mogi M, Horiuchi M. Clinical Interaction between Brain and Kidney in Small Vessel Disease. Cardiol Res Pract. 2011 Jan;2011:306189.

22 Dweck MR, Boon NA, Newby DE. Calcific aortic stenosis: a disease of the valve and the myocardium. J Am Coll Cardiol. 2012 Nov;60(19):1854-63.

23 Toyoda K, Ninomiya T. Stroke and cerebrovascular diseases in patients with chronic kidney disease. Lancet Neurol. 2014 Aug;13(8):823-33.

24 Boon A, Lodder J, Cheriex E, Kessels F. Risk of stroke in a cohort of 815 patients with calcification of the aortic valve with or without stenosis. Stroke. 1996 May;27(5):847-51.

25 Rodriguez CJ, Bartz TM, Longstreth WT Jr, Kizer JR, Barasch E, Lloyd-Jones DM, et al. Association of annular calcification and aortic valve sclerosis with brain findings on magnetic resonance imaging in community dwelling older adults: the cardiovascular health study. J Am Coll Cardiol. 2011 May;57(21):2172-80. 